\title{
Cobalt-catalyzed C-H cyanations: Insights into the reaction mechanism and the role of London dispersion
}

\author{
Eric Detmar ${ }^{1}$, Valentin Müller ${ }^{2}$, Daniel Zell ${ }^{2}$, Lutz Ackermann ${ }^{* 2}$ and Martin Breugst ${ }^{* 1}$
}

\author{
Full Research Paper \\ Address: \\ ${ }^{1}$ Department für Chemie, Universität zu Köln, Greinstraße 4, 50939 \\ Köln, Germany and ${ }^{2}$ Institut für Organische und Biomolekulare \\ Chemie, Georg-August-Universität Göttingen, Tammannstraße 2, \\ 37077 Göttingen, Germany

\section{Email:} \\ Lutz Ackermann* - Lutz.Ackermann@chemie.uni-goettingen.de; \\ Martin Breugst ${ }^{*}$ - mbreugst@uni-koeln.de. \\ * Corresponding author \\ Keywords: \\ catalysis; $\mathrm{C}-\mathrm{H}$ activation; density functional theory; London \\ dispersion; reaction mechanisms
}

Beilstein J. Org. Chem. 2018, 14, 1537-1545.

doi:10.3762/bjoc. 14.130

Received: 27 February 2018

Accepted: 02 June 2018

Published: 25 June 2018

This article is part of the Thematic Series "Dispersion interactions".

Guest Editor: P. Schreiner

(C) 2018 Detmar et al.; licensee Beilstein-Institut.

License and terms: see end of document.

\begin{abstract}
Carboxylate-assisted cobalt(III)-catalyzed C-H cyanations are highly efficient processes for the synthesis of (hetero)aromatic nitriles. We have now analyzed the cyanation of differently substituted 2-phenylpyridines in detail computationally by density functional theory and also experimentally. Based on our investigations, we propose a plausible reaction mechanism for this transformation that is in line with the experimental observations. Additional calculations, including NCIPLOT, dispersion interaction densities, and local energy decomposition analysis, for the model cyanation of 2-phenylpyridine furthermore highlight that London dispersion is an important factor that enables this challenging $\mathrm{C}-\mathrm{H}$ transformation. Nonbonding interactions between the $\mathrm{Cp} *$ ligand and aromatic and $\mathrm{C}-\mathrm{H}$-rich fragments of other ligands at the cobalt center significantly contribute to a stabilization of cobalt intermediates and transition states.
\end{abstract}

\section{Introduction}

For a long time, large and bulky substituents have intuitively been considered to act through unfavorable steric interactions, although London dispersion - the attractive part of the van-derWaals interaction - is known for more than 100 years [1,2]. The stabilizing nature of $\mathrm{C}-\mathrm{H} \cdots \mathrm{H}-\mathrm{C}$ interactions and their impor- tance for organic transformations has only been fully realized within the last decades [3]. Among others, these interactions explain the hexaarylethane riddle [4] and are responsible for the high stability of singly bonded diamondoid dimers resulting in very long $\mathrm{C}-\mathrm{C}$ bonds $[5,6]$, or very short $\mathrm{H} \cdots \mathrm{H}$ contacts in 
tris(3,5-di-tert-butylphenyl)methane [7]. Besides a remarkable effect on organic structures, dispersion can also affect the outcome of chemical transformations. Presumably due to attractive dispersive interactions between two adamantyl groups in the transition state of a $[4+2]$ cycloaddition of benzynes (Scheme 1), the seemingly sterically more hindered product is formed preferentially [8].

Similar to other noncovalent interactions [9-11], London dispersion can also play a crucial role in different transition-metal-catalyzed reactions [12-17]. The C-H-rich di-1-adamantylphosphine oxide - a typical dispersion element - was experimentally found to be an excellent preligand for ruthenium- and palladium-catalyzed $\mathrm{C}-\mathrm{H}$ functionalizations [18-23]. Similarly, computational studies revealed the importance of dispersion effects in palladium-catalyzed cross-coupling reactions [24-27]. For example, the contribution of London dispersion (up to $37 \mathrm{kcal} \mathrm{mol}^{-1}$ ) has a huge influence on the ligand dissociation process within the $\mathrm{Pd}\left(\mathrm{PPh}_{3}\right)_{4}$ system [25]. Furthermore, only the results obtained from dispersion-corrected density functional theory $[28,29]$ were in agreement with the experimental observations and dispersion reduces the activation free energies by up to $30 \mathrm{kcal} \mathrm{mol}^{-1}$ [27].

Currently, the strategic application of London dispersion in catalysis is still very difficult to achieve and, as a consequence, detailed insights in how dispersion influences organic reactions continue to be in high demand. Therefore, we have computationally analyzed the recently developed cobalt-catalyzed $\mathrm{C}-\mathrm{H}$ cyanation of arenes (Scheme 2) [30-34]. Dispersion effects can be envisioned to be highly important in this system, as the relatively $\mathrm{C}-\mathrm{H}$-rich ligand $\mathrm{Cp}$ * can interact with both substrates within the cobalt complexes. In 2015, Li and Ackermann have proposed the catalytic cycle ( $\mathrm{C}-\mathrm{H}$ cobaltation, ligand coordination, insertion) shown in Scheme 2 which served as the starting point of this investigation [30]. We now report on our computational findings supported by novel kinetic investigations to establish the reaction mechanism of this synthetically useful $\mathrm{C}-\mathrm{H}$ activation and to elucidate the role of London dispersion in these transformations.

\section{Results and Discussion Analysis of the reaction mechanism}

To unravel the importance of London dispersion on the cobaltcatalyzed C-H cyanation of 2-phenylpyridine (1a), the underlying catalyst's mode of action has to be fully understood. The available experimental data indicated a reversible $\mathrm{C}-\mathrm{H}$ metalation, which led to the suggested catalytic cycle of Scheme 2 [30]. As computational investigations also allow the study of intermediates that are too unstable to be observed under the experimental conditions, we have analyzed the underlying reaction mechanism in more detail employing density functional theory. A complete free energy profile on the B3LYP-D3BJ/ def2-QZVP/COSMO//B3LYP-D3BJ/def2-TZVP potential energy surface is depicted in Figure 1 (black line), while the free-energy profile on the M06-L surface is summarized in Supporting Information File 1. Selected intermediates and transition states are shown in Figure 2.

The computational analysis starts with the catalytically active cobalt(III) acetate complex $\mathbf{4}$ which is generated in situ from the precatalyst $\left[\mathrm{Cp}^{*} \mathrm{CoI}_{2}(\mathrm{CO})\right], \mathrm{AgSbF}_{6}$, and $\mathrm{KOAc}$. While the iodine ions are captured by $\mathrm{Ag}^{+}$, carbon monoxide dissociates and leaves the reaction mixture as a gas. Although the $\mathrm{SbF}_{6}{ }^{-}$ counter ion to the cationic cobalt complexes is considered to be weakly coordinating [37], specific interactions cannot be completely ruled out. We have assumed that all of the positively charged cobalt complexes on the reaction path are similarly affected by ion pairing and therefore, we base the following investigation mainly on the reactions of the cobalt complexes and do not include ion pairing in our analysis. Coordination of 2-phenylpyridine (1a) to this 16-electron species leads to the intermediate 5a (Figure 2) in a highly exergonic reaction step ( $\Delta G=-18.8 \mathrm{kcal} \mathrm{mol}^{-1}$ ), which also is the resting state of the catalytic cycle. This intermediate could therefore be amenable to spectroscopic characterization. Based on our computational analysis, the subsequent $\mathrm{C}-\mathrm{H}$ cobaltation $(\mathbf{5 a} \rightarrow \mathbf{7 a})$ is endergonic $\left(\Delta \Delta G=+12 \mathrm{kcal} \mathrm{mol}^{-1}\right)$ and proceeds in a step-wise fashion. A similar mechanism has previously been described by McMullin, Williams, and Frost [38], as well as by Ackermann $[39,40]$ for ruthenium-catalyzed $\mathrm{C}-\mathrm{H}$ alkenylations. In the first

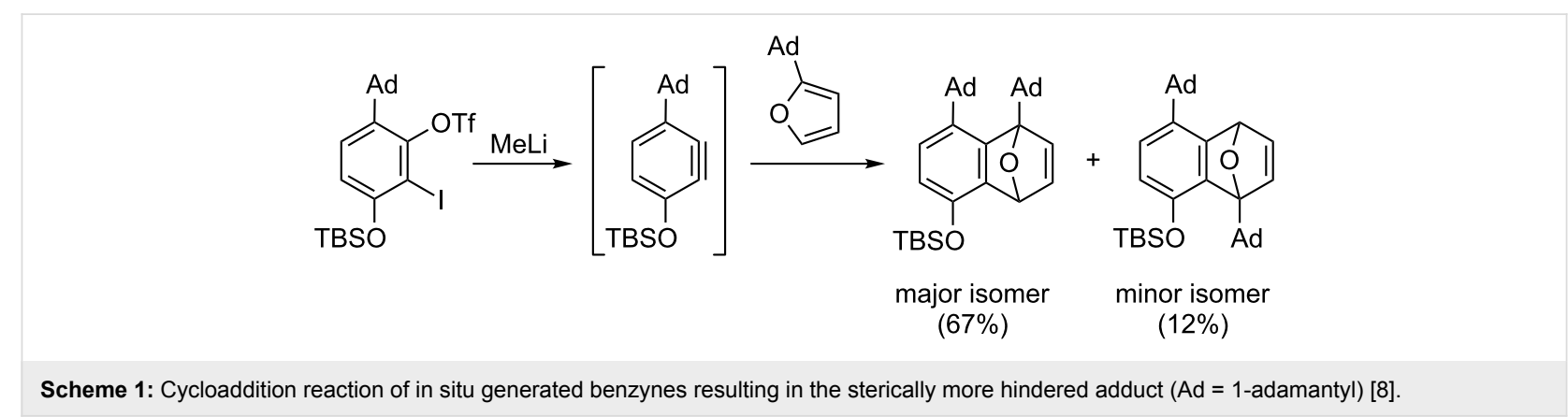


<smiles>c1ccc(-c2ccccn2)cc1</smiles>

1 a

\section{$\mathrm{Ts}_{\substack{\mathrm{N}^{-} \\ \mathrm{C}}}^{\mathrm{C}}$}

$2 a$
$\left[\mathrm{Cp}^{*} \mathrm{Col}_{2}(\mathrm{CO})\right](2.5 \mathrm{~mol} \%)$ $\mathrm{AgSbF}_{6}(5.0 \mathrm{~mol} \%)$ $\operatorname{KOAC}(5.0 \mathrm{~mol} \%)$

DCE, $120^{\circ} \mathrm{C}, 16 \mathrm{~h}$<smiles>N#Cc1ccccc1-c1ccccn1</smiles>

$3 \mathbf{a}$

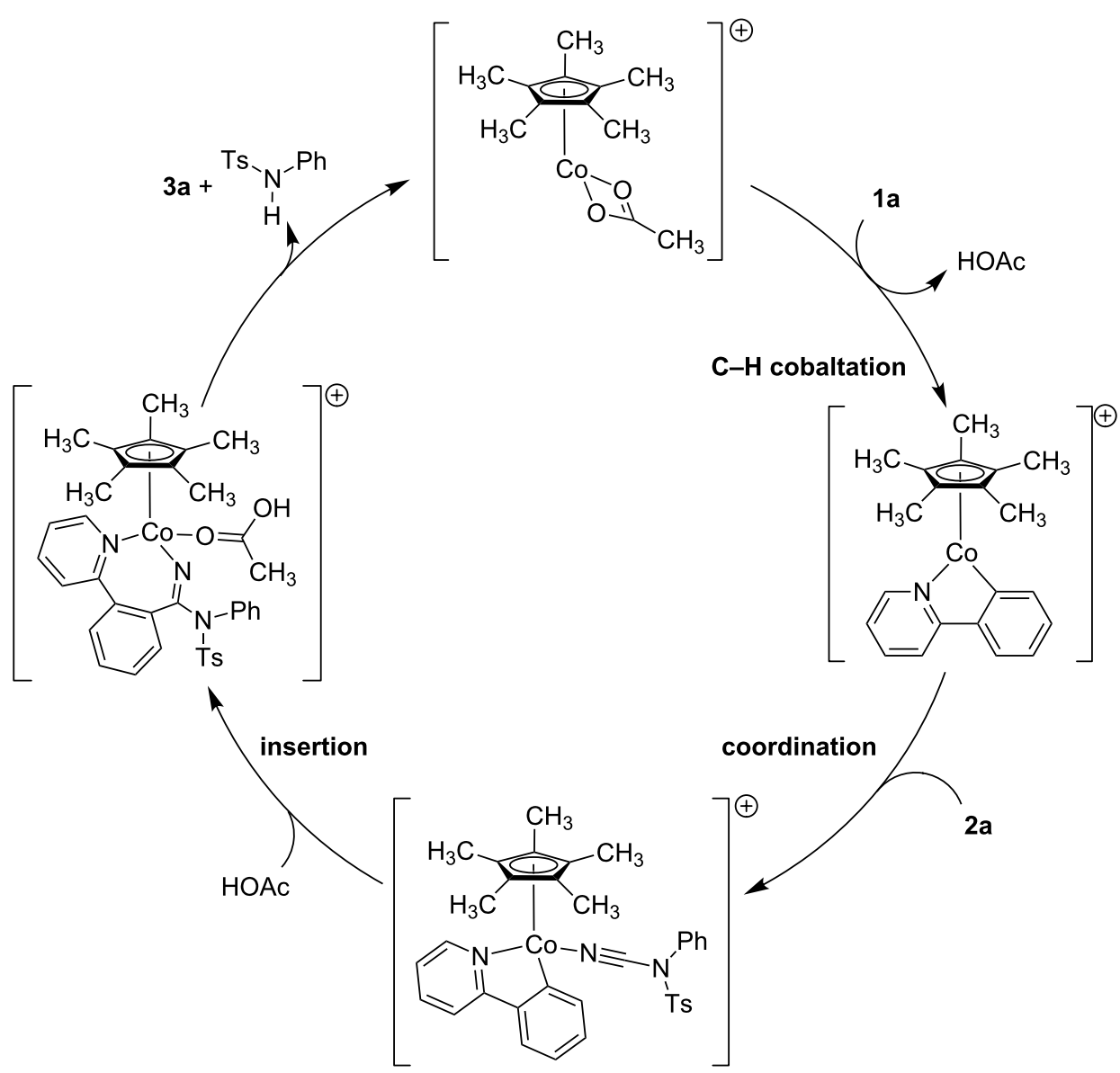

Scheme 2: Recently developed cobalt-catalyzed C-H cyanation [30].

transition state (TS1a, Figure 2), the $\kappa^{2}$-coordination of the acetate ligand changes to a $\kappa^{1}$-coordination. The resulting intermediate 6a is stabilized by an agostic interaction between the $\mathrm{C}-\mathrm{H}$ bond and the metal atom as well as by an additional weak hydrogen bond between the $\mathrm{C}-\mathrm{H}$ bond and the acetate oxygen $(\mathrm{O} \cdots \mathrm{H}$ distance $2.26 \AA)$. A natural population analysis of structure 6a further confirms the stabilizing nature of these interactions. In the second transition state TS2a (Figure 2), the $\mathrm{C}-\mathrm{H}$ bond is broken and the proton is transferred to the acetate which results in the formation of the cobaltacycle 7a.

Acetic acid dissociates, and $N$-cyano- $N$-phenyl- $p$-toluenesulfonamide (2a) coordinates to the 16-electron intermediate 8a yielding 9a. Next, the insertion of the cyanating agent $\mathbf{2 a}$ into the cobalt-carbon bond takes place through TS3a. Within the four-membered transition state (Figure 2), the $\mathrm{C}-\mathrm{C}$ bond to be formed is still rather long ( $\mathrm{C}-\mathrm{C}$ distance $1.92 \AA$ ), while the $\mathrm{C}-\mathrm{N}$ distance is already significantly elongated $(1.15 \AA$ in $9 \mathrm{a}, 1.22 \AA$ in TS3a, $1.26 \AA$ in 10a). Furthermore, a significant reorganization has to take place during this step: the former almost linear $\mathrm{N}-\mathrm{C}-\mathrm{N}$ fragment $\left(179.2^{\circ}\right)$ changes to $137.9^{\circ}$ in TS3a and $124.4^{\circ}$ in $\mathbf{1 0 a}$, which results in a high barrier for this step. Subsequent coordination of acetic acid leads to intermediate 11a. No transition states could be obtained for the following $\beta$-elimination and proto-demetalation resulting in product 3a, the cobalt(III) acetate complex 4 , and $N$-phenyl-p-toluenesulfonamide. All attempts starting from different potential transition state structures resulted in barrierless reactions 

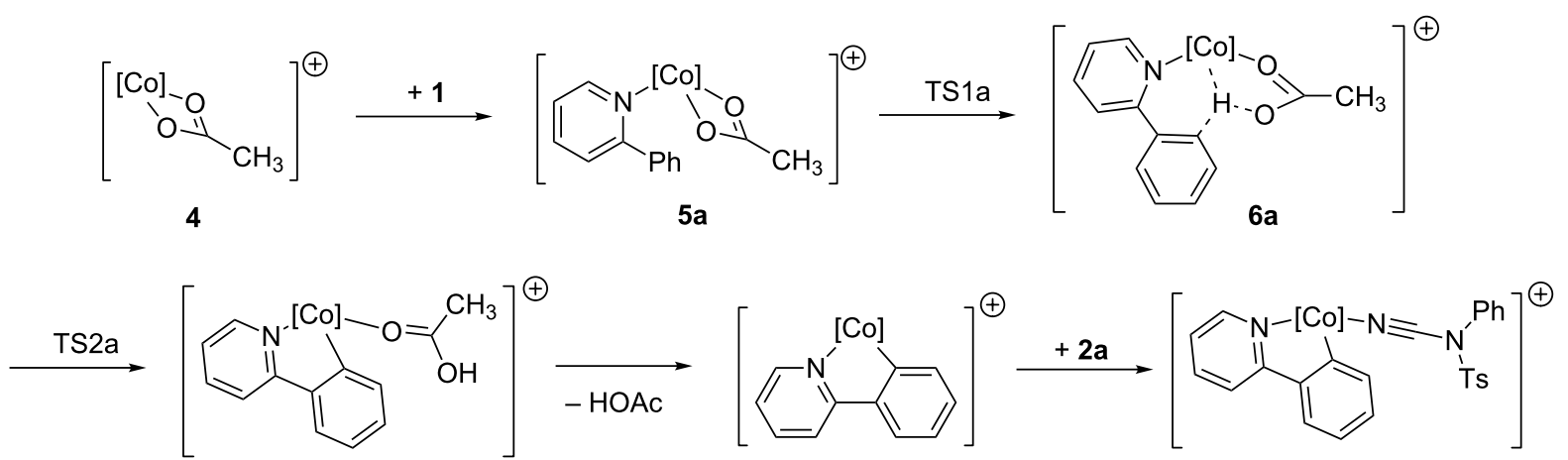

$7 \mathbf{a}$

$8 a$

$9 a$

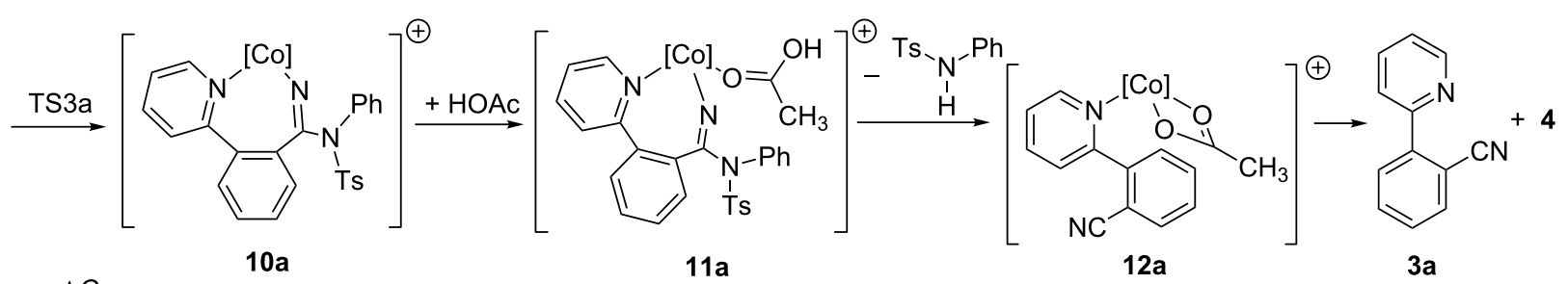

$\Delta G$

$11 \mathrm{a}$

$12 a$

$3 a$

[kcal mol $\mathrm{mo}^{-1}$

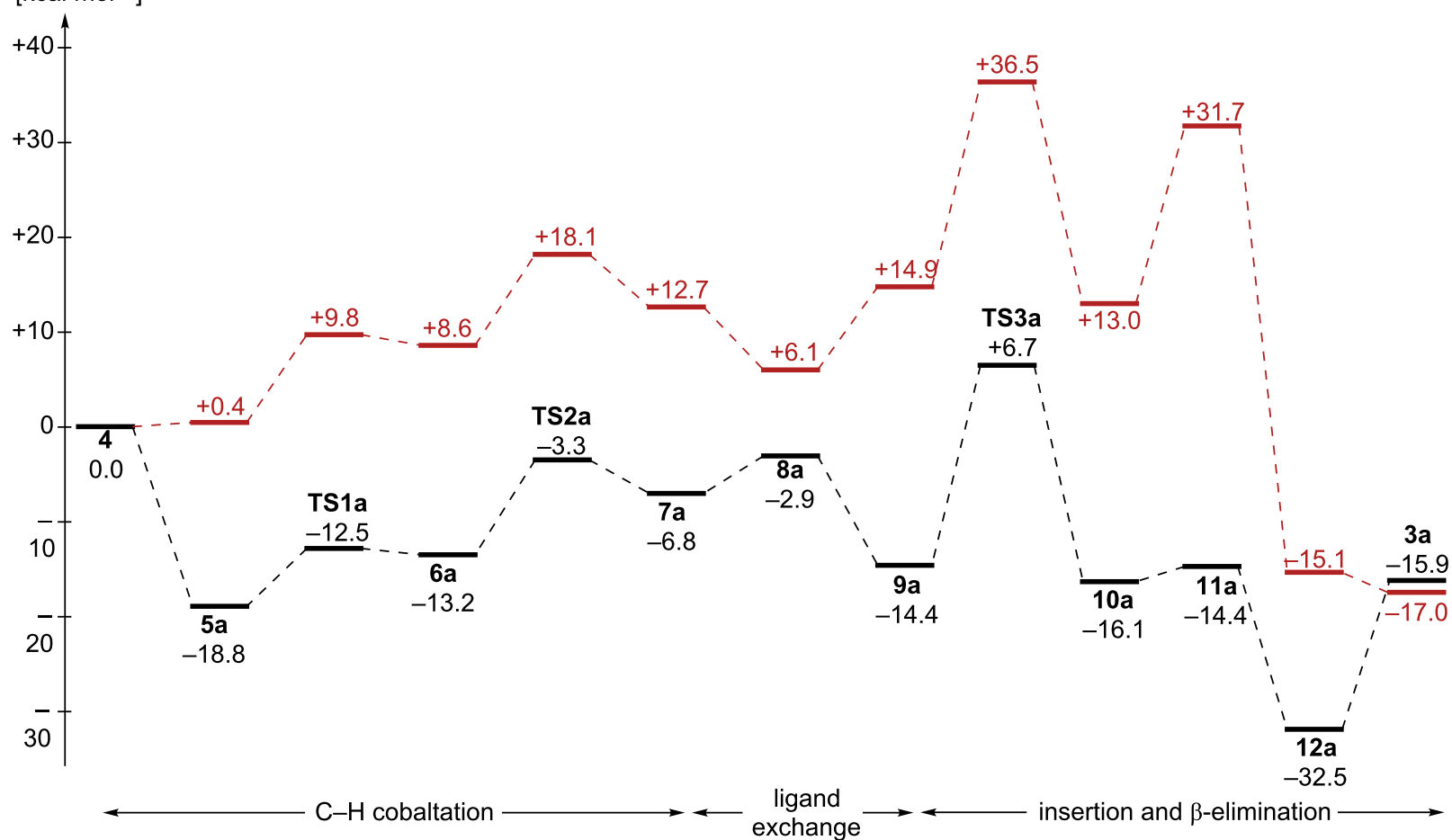

Figure 1: Calculated free-energy profile for the cobalt-catalyzed $\mathrm{C}-\mathrm{H}$ cyanation of 2-phenylpyridine (1a) [in $\mathrm{kcal}^{\mathrm{mol}}{ }^{-1},[\mathrm{Co}]=\mathrm{Cp}{ }^{*} \mathrm{Co}$, black lines indicate that dispersion (D3 correction with Becke-Johnson damping) $[35,36]$ was included in the calculations while red lines indicate that dispersion was not included].

when a proton approaches the amidine substructure $(\rightarrow \mathbf{1 2 a})$. As the cyanated 2-phenylpyridine $\mathbf{3 a}$ is less Lewis-basic compared to the starting material 1a, 12a could also react with $\mathbf{1 a}$ in a thermodynamically favorable ligand exchange reaction $\left(\Delta G=-2.2 \mathrm{kcal} \mathrm{mol}^{-1}\right)$ to yield complex $\mathbf{5 a}$.
In contrast to previous computational studies on manganese(I)catalyzed fluoro-allylation reactions where $\beta$-fluoride and HF eliminations played an important role [41], similar reactions involving amine eliminations seem to be not relevant in this reaction. Furthermore, a comparison with previous computational 


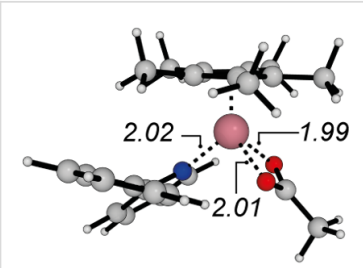

$5 a$

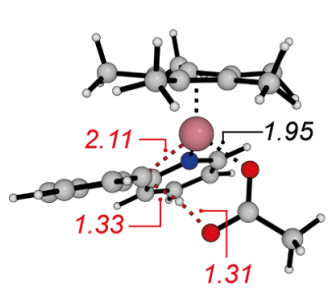

TS2a

$\left(1123 \mathrm{icm}^{-1}\right)$

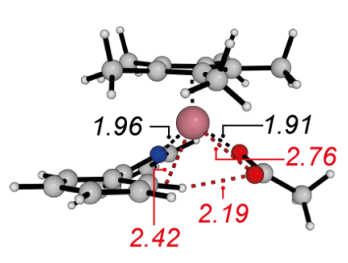

TS1a

$\left(64 \mathrm{icm}^{-1}\right)$

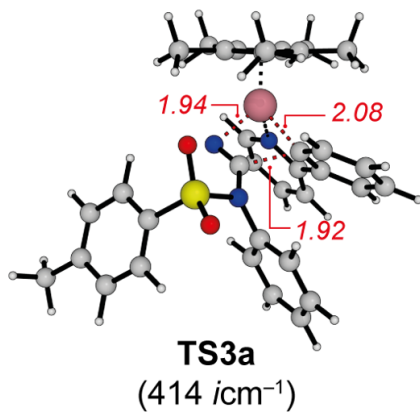

$\left(414 \mathrm{icm}^{-1}\right)$
Figure 2: Calculated structures, selected bond lengths (in $\AA$ ), and imaginary frequencies for representative intermediates and transition states for the cobalt-catalyzed C-H cyanation of 2-phenylpyridine (1a)

investigations on copper-catalyzed ortho $\mathrm{C}-\mathrm{H}$ cyanations of vinylarenes revealed that those reactions take place via a completely different mechanism involving two distinct catalytic cycles (copper-catalyzed electrophilic cyanative dearomatization and base-catalyzed hydrogen transposition) $[42,43]$.

Inspired by this computational analysis, we experimentally probed the effect of differently substituted cyanation agents 2 on the kinetics of the cobalt(III)-catalyzed $\mathrm{C}-\mathrm{H}$ cyanation (Scheme 3). Thus, we observed that electron-withdrawing groups significantly facilitated the desired transformation. As the calculated rate-limiting transition state TS3 benefits from a stabilization of the developing negative charge on the sulfonamide, the relative rates of Scheme 3 provide further support for the migratory insertion representing the rate determining step [44].

As differently substituted 2-phenylpyridines 1 have been employed experimentally, we included five representative substrates $\left(\mathrm{R}=\mathrm{H}, \mathrm{CH}_{3}, \mathrm{~F}, \mathrm{C}(\mathrm{O}) \mathrm{CH}_{3}, \mathrm{CN}\right)$ into the computational analysis as well. For these calculations, only one functional (B3LYP-D3BJ) and a smaller basis set (def2-SVP for nonmetals and def2-TZVP for Co) were employed during the optimization to reduce the computational cost. These results are summarized in Table 1.

For the unsubstituted 2-phenylpyridine (1a), both computational methods (Figure 1 and Table 1) and the optimized structures are generally rather similar to one another. Based on the
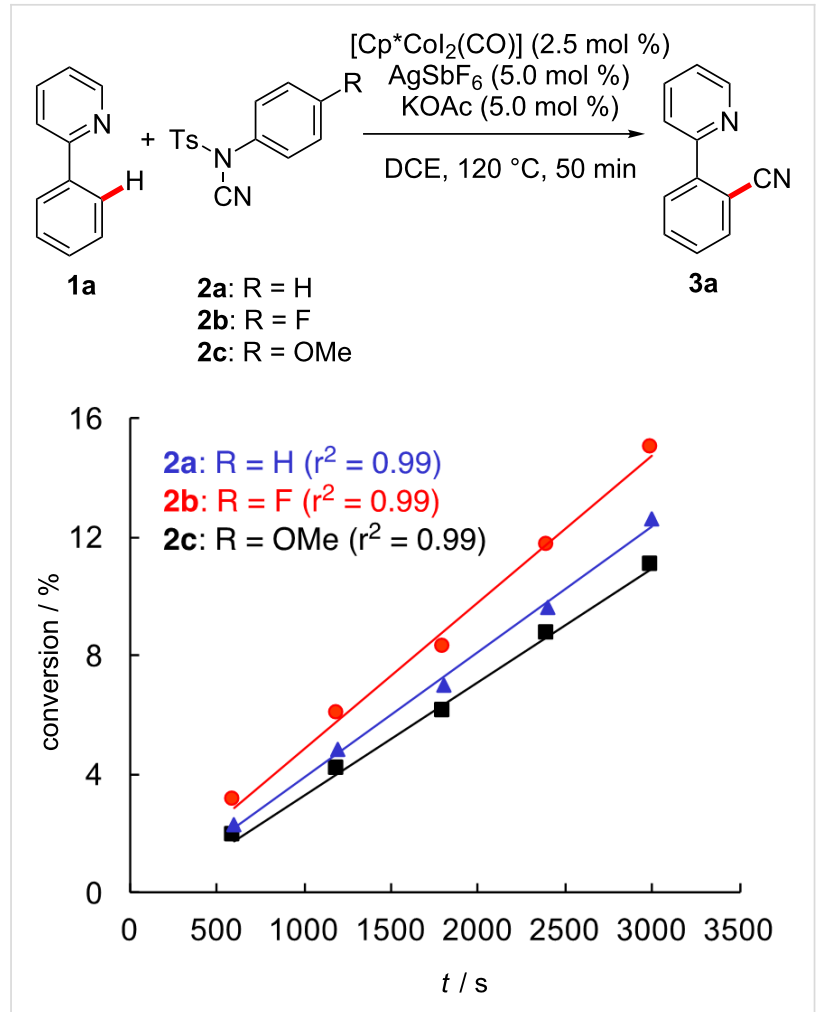

Scheme 3: Kinetic profile of the cobalt-catalyzed C-H cyanation with differently substituted cyanating agents 2 .

computational analysis depicted in Table 1, the turnoverlimiting step for all substrates $\mathbf{1}$ is represented by the insertion of the cyanating agent $\mathbf{2 a}$ into the cobalt-carbon bond, which can also be concluded based on the kinetic data of Scheme 3 .

Based on the computational analysis of Figure 1 and the experimental data depicted in Scheme 2, the turnover-limiting step for this transformation is the insertion of $\mathbf{2 a}$ with an overall barrier of $25.5 \mathrm{kcal} \mathrm{mol}^{-1}$. The initial $\mathrm{C}-\mathrm{H}$ cobaltation occurs with a smaller activation free energy of $15.5 \mathrm{kcal} \mathrm{mol}^{-1}$. These values are also in good qualitative agreement with the experimental findings: The calculated high barriers match the prolonged reaction times and high temperature required in the experimental studies and the reversible $\mathrm{C}-\mathrm{H}$ metalation [30].

\section{Influence of London dispersion}

In recent years, London dispersion, the attractive part of the van-der-Waals force, has been repeatedly identified as key to stabilizing organic structures and facilitating novel reactivities [3]. As the $\mathrm{Cp}^{*}$ ligand is a $\mathrm{C}-\mathrm{H}-$ rich molecule, we envisioned that dispersive interactions should be important for this transformation as well. As a consequence, we have analyzed this reaction additionally with B3LYP without dispersion correction and the dispersion-corrected M06-L functional under otherwise identical conditions as a first starting point. Independent of the 
Table 1: Calculated free energies for the reaction mechanism involving differently substituted 2-phenylpyridines 1a-e [B3LYP-D3BJ/def2-QZVP/ COSMO//B3LYP-D3BJ/def2-SVP, def2-TZVP for Co].

\begin{tabular}{|c|c|c|c|c|c|c|c|c|c|c|c|c|c|}
\hline & & $1 a-e$ & & $2 a$ & & & & & $3 a-$ & & & & \\
\hline & 4 & 5 & TS1 & 6 & TS2 & 7 & 8 & 9 & TS3 & 10 & 11 & 12 & 3 \\
\hline $\mathrm{R}=\mathrm{H}(\mathbf{1} \mathrm{a})$ & 0.0 & -20.1 & -12.4 & -14.1 & -5.4 & -8.4 & -4.2 & -15.5 & +3.4 & -18.3 & -16.0 & -34.5 & -18.0 \\
\hline $\mathrm{R}=\mathrm{CH}_{3}(\mathbf{1 b})$ & 0.0 & -21.0 & -14.2 & -15.7 & -7.1 & -9.6 & -4.5 & -18.5 & +0.6 & -18.8 & -16.1 & -35.5 & -18.9 \\
\hline$R=F(1 c)$ & 0.0 & -19.8 & -12.0 & -12.4 & -4.8 & -9.7 & -4.4 & -17.5 & +4.5 & -17.3 & -14.9 & -32.3 & -16.8 \\
\hline $\mathrm{R}=\mathrm{C}(\mathrm{O}) \mathrm{CH}_{3}(\mathbf{1 d})$ & 0.0 & -18.1 & -9.6 & -11.2 & -3.4 & -7.4 & -2.1 & -17.7 & +3.4 & -17.5 & -13.2 & -30.9 & -16.4 \\
\hline $\mathrm{R}=\mathrm{CN}(\mathbf{1 e})$ & 0.0 & -18.0 & -9.9 & -9.9 & -3.1 & -8.9 & -3.5 & -16.8 & +5.4 & -14.9 & -12.4 & -29.4 & -15.5 \\
\hline
\end{tabular}

computational method, the overall reaction free energy for the transformation of Scheme 2 is almost identical [-15.9 (B3LYPD3BJ), -17.0 (B3LYP), and -15.9 (M06-L) kcal mol ${ }^{-1}$ ] indicating that dispersion is less important for the overall thermodynamics of this reaction. In contrast, a strong effect of the functional was observed for the complete energy profile. While the dispersion-corrected functional M06-L (see the Supporting Information File 1 for details) resulted in a comparable profile to that obtained with B3LYP-D3BJ (black lines in Figure 1), a significant deviation was observed when the latter was used without any dispersion correction (red lines in Figure 1). All cobalt complexes are substantially stabilized by dispersive interactions resulting in a significant net reduction of the activation free energy by $11 \mathrm{kcal} \mathrm{mol}^{-1}$. Comparable contributions of London dispersion have also been calculated with other functionals (TPSS [45] and PBE [46,47]). As expected, complexes with more nonbonding contacts (e.g., 10a) are better stabilized than complexes where the $\mathrm{Cp}^{*}$ ligand is located farther away from other ligands (e.g., 8a). In comparison to computational investigations of Pd-catalyzed reactions [27], similar dispersive stabilizations of individual complexes have been calculated here.

A closer qualitative analysis of the intramolecular interactions in these complexes employing the NCIPLOT program $[48,49]$ furthermore confirms these noncovalent interactions. While all plots are shown in Supporting Information File 1, Figure 3 summarizes those for selected intermediates and transition states. For all structures, significant interactions can be found between the $\mathrm{Cp}$ * ligand and the various phenyl groups of the reagents. In addition, the presence of additional stabilizing interactions such as further hydrogen bonds can also be confirmed by this analysis (e.g., in TS3, see also the Supporting Information File 1).

To further probe the dispersive interaction of the $\mathrm{Cp}^{*}$ ligand and the other ligands, we have additionally calculated the dispersion interaction densities (DID) [50] for all intermediates and transition states at the SCS-LMP2/def2-TZVPP level of theory. The DID plots of Figure 4 reveal that medium to strong

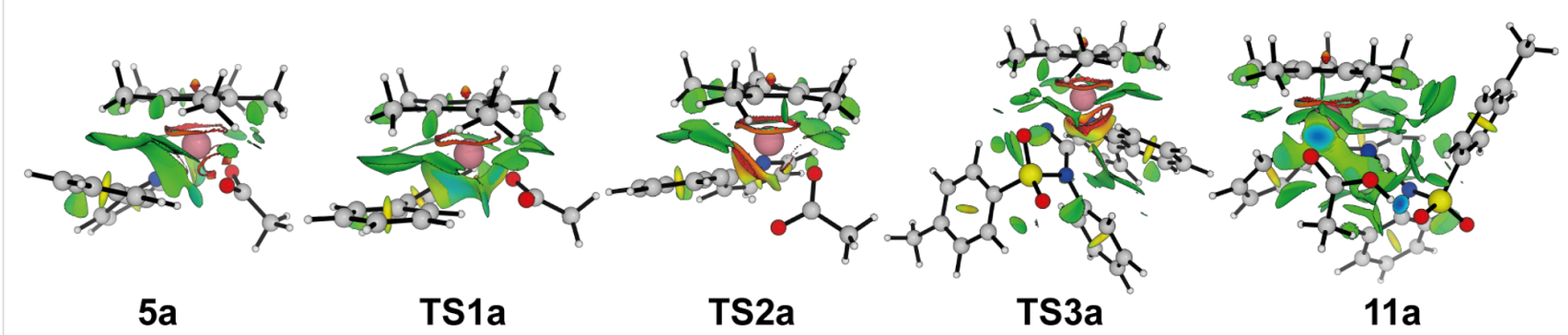

Figure 3: Noncovalent interaction $(\mathrm{NCl})$ analysis for selected intermediates and transition states. The gradient isosurfaces $(s=0.5$ au $)$ are colored according to the sign of $\left(\lambda_{2}\right) \rho$ over the range of -0.05 (blue) to +0.05 (red). 


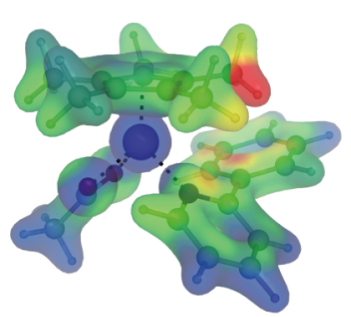

$5 a$

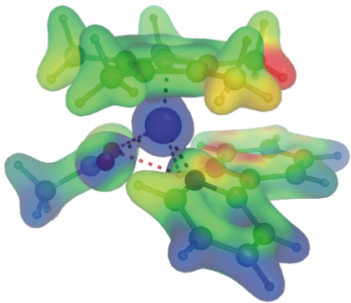

TS1a

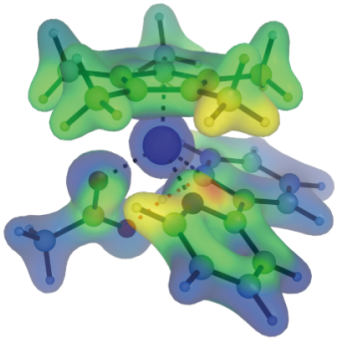

TS2a

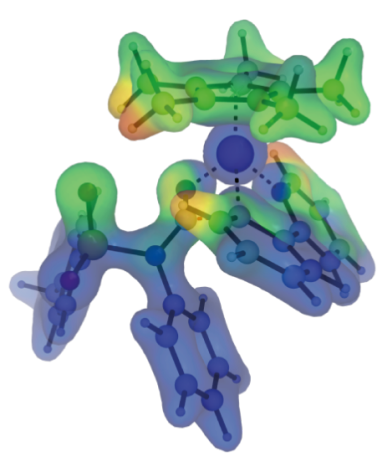

TS3a

$$
\begin{aligned}
& \text { London Dispersion } \\
& \text { none strong }
\end{aligned}
$$

Figure 4: Projected dispersion interaction density (DID) plots for selected intermediates and transition states. The molecular density isosurfaces $\left(0.1 \mathrm{e} / \mathrm{Bohr}^{3}\right)$ are colored from zero interaction energy (blue) to the strongest dispersion interaction (red).

dispersive interactions can be found between the $\mathrm{Cp}^{*}$ ligand and the aromatic and $\mathrm{C}-\mathrm{H}$-rich fragments in its proximity. In line with the analyses presented in Figure 3 and Figure 4, a local energy decomposition (LED) analysis [51] using DLPNO$\operatorname{CCSD}(\mathrm{T}) / \mathrm{cc}-\mathrm{pVDZ}$ also confirmed medium to strong dispersive interactions up to $12 \mathrm{kcal} \mathrm{mol}^{-1}$ between the $\mathrm{Cp}^{*}$ ligand and the other ligands. Based on the computational analysis, London dispersion is not only highly beneficial for the synthetically important cobalt-catalyzed $\mathrm{C}-\mathrm{H}$ cyanation reaction, but it also emphasises that the $\mathrm{Cp}^{*}$ ligand does not exclusively act as a sterically demanding ligand in transition-metal-catalyzed reactions.

\section{Conclusion}

We have analyzed the cobalt(III)-catalyzed $\mathrm{C}-\mathrm{H}$ cyanation of differently substituted 2-phenylpyridines with $\mathrm{N}$-cyano- $\mathrm{N}$-aryl$p$-toluenesulfonamide using density functional theory. On the basis of our computational and experimental data, we can propose a reaction mechanism for this transformation. After an initial and reversible $\mathrm{C}-\mathrm{H}$ cobaltation, the subsequent insertion of the cyanating agents is the rate-limiting step. In addition, our calculations unravel that all the cobalt intermediates are considerably affected by London dispersion, which also results in a significant stabilization of the rate-limiting transition state.

\section{Computational Details}

For all structures, geometry optimizations were performed with three different functionals using the def2-TZVP (def2-TZVPP for M06-L) basis set [52] and the $\mathrm{m} 4$ numerical quadrature grid in the gas phase. The hybrid functional B3LYP $[53,54]$ with and without Grimme's dispersion correction D3 (Becke-Johnson damping) $[35,36]$ as well as Truhlar's dispersion-corrected M06-L [55] functional were employed in this investigation. For the latter, the density fitting RI-J approach was used to accel- erate the calculations [56,57]. For the analysis of the substituent effect, the B3LYP functional with Grimme's dispersion correction D3 (Becke-Johnson damping) was employed together with the def2-SVP basis set for all non-metals and the def2-TZVP basis set for Co. Vibrational analysis verified that each structure was a minimum or transition state $\left(i \omega<30 \mathrm{~cm}^{-1}\right.$ were tolerated). Thermal corrections were calculated from unscaled harmonic vibrational frequencies at the same levels of theory and refer to a standard state of $298.15 \mathrm{~K}$ and $1 \mathrm{~mol} \mathrm{~L}^{-1}$. Entropic contributions to the reported free energies were obtained from partition functions evaluated with Truhlar's quasiharmonic approximation [58]. This method uses the same approximations as the usual harmonic oscillator approximation except that all vibrational frequencies lower than $100 \mathrm{~cm}^{-1}$ are set equal to $100 \mathrm{~cm}^{-1}$. Energies were subsequently derived from single-point calculations employing the functionals described above, the quadruple- $\zeta$ basis set def2-QZVP [52] and the COSMO solvation model [59] for dichloroethane $(\varepsilon=10.125)$. The dispersion interaction densities (DID) [50] were calculated at the SCS-LMP2/def2-TZVPP level of theory using MOLPRO $2015[60,61]$. The local energy decomposition analysis [51] was performed employing Neese's domain-based local pair-natural orbital (DLPNO) approach to the CCSD(T) method [DLPNO$\operatorname{CCSD}(\mathrm{T})$ ] [62-64] with tightPNO settings and the double- $\zeta$ cc-pVDZ basis set as implemented in ORCA 4 [65]. All DFT calculations were performed with Turbomole $7.1[66,67]$ and the NCIPLOT code was employed for the visualization noncovalent interactions $[48,49]$.

\section{Experimental Details}

General remarks: Catalytic reactions were carried out in Schlenk flasks under nitrogen atmosphere using predried glassware. 1,2-Dichlorethane (DCE) was dried and distilled over $\mathrm{CaH}_{2}$ under $\mathrm{N}_{2} . N$-Cyano- $N$-phenyl- $p$-toluenesulfonamide (2a) 
[68] and $\mathrm{Cp}^{*} \mathrm{Co}(\mathrm{CO}) \mathrm{I}_{2}[69]$ were synthesized according to previously described methods. Other chemicals were obtained from commercial sources and were used without further purification.

Kinetic experiments of the cobalt(III)-catalyzed $\mathbf{C}-\mathbf{H}$ cyanation: A suspension of 1 (78 $\mathrm{mg}, 0.50 \mathrm{mmol}), 2(0.75 \mathrm{mmol})$, $\left[\mathrm{Cp} * \mathrm{Co}(\mathrm{CO}) \mathrm{I}_{2}\right](6.0 \mathrm{mg}, 2.5 \mathrm{~mol} \%), \mathrm{AgSbF}_{6}(8.6 \mathrm{mg}$, $5.0 \mathrm{~mol} \%)$ and KOAc $(2.5 \mathrm{mg}, 5.0 \mathrm{~mol} \%)$ in DCE $(2.0 \mathrm{~mL})$ was heated at $120{ }^{\circ} \mathrm{C}$. Aliquots up to ca $15 \%$ conversion $(25 \mu \mathrm{L} ; 10,20,30,40,50 \mathrm{~min})$ were periodically removed by a syringe and directly analyzed by GC using $n$-dodecane $(30 \mu \mathrm{L})$ as internal standard.

2-(Pyridin-2-yl)benzonitrile (3a): ${ }^{1} \mathrm{H} \mathrm{NMR}\left(\mathrm{CDCl}_{3}\right.$, $400 \mathrm{MHz}) \delta 8.73-8.70(\mathrm{ddd}, J=4.7,1.8,0.9 \mathrm{~Hz}, 1 \mathrm{H})$, $7.82-7.71(\mathrm{~m}, 4 \mathrm{H}), 7.64$ (dd, $J=7.6,1.4 \mathrm{~Hz}, 1 \mathrm{H}), 7.49$ (dd, $J=$ 7.6, $1.2 \mathrm{~Hz}, 1 \mathrm{H}), 7.31$ (ddd, $J=7.4,4.7,1.2 \mathrm{~Hz}, 1 \mathrm{H}) ;{ }^{13} \mathrm{C} \mathrm{NMR}$ $\left(\mathrm{CDCl}_{3}, 125 \mathrm{MHz}\right) \delta 155.1\left(\mathrm{C}_{\mathrm{q}}\right), 149.8(\mathrm{CH}), 143.4\left(\mathrm{C}_{\mathrm{q}}\right), 136.7$ $(\mathrm{CH}), 134.0(\mathrm{CH}), 132.7(\mathrm{CH}), 129.9(\mathrm{CH}), 128.6(\mathrm{CH}), 123.2$ $(\mathrm{CH}), 123.1(\mathrm{CH}), 118.6\left(\mathrm{C}_{\mathrm{q}}\right), 111.0\left(\mathrm{C}_{\mathrm{q}}\right)$; IR (ATR): 3350, $2224,1560,1464,758,509 \mathrm{~cm}^{-1}$. EIMS $\mathrm{m} / z$ (relative intensity): 180 (100) $\left[\mathrm{M}^{+}\right], 154$ (5), 140 (5), 126 (5), 102 (5), 75 (5); HRMS (EI) $m / z$ : $\left[\mathrm{M}^{+}\right]$calcd. for $\mathrm{C}_{12} \mathrm{H}_{8} \mathrm{~N}_{2}, 180.0687$; found, 180.0684. The analytical data are in accordance with those reported in literature [30].

\section{Supporting Information}

\section{Supporting Information File 1 \\ Cartesian coordinates, energies of all calculated structures, and details of computational methods. \\ [https://www.beilstein-journals.org/bjoc/content/ supplementary/1860-5397-14-130-S1.pdf]}

\section{Acknowledgements}

Financial support from the Fonds der chemischen Industrie (Liebig scholarship to M.B.), the DFG (SPP 1807), and from the University of Cologne within the excellence initiative is gratefully acknowledged. We are very grateful to Professor Ricardo Mata and Axel Wuttke for help with the calculation of dispersion interaction densities. We thank the Regional Computing Center of the University of Cologne (RRZK) for providing computing time on the DFG-funded High Performance Computing (HPC) system CHEOPS as well as for their support.

\section{References}

1. London, F. Z. Phys. 1930, 63, 245-279. doi:10.1007/BF01421741

2. London, F. Trans. Faraday Soc. 1937, 33, 8b-26.

doi:10.1039/tf937330008b
3. Wagner, J. P.; Schreiner, P. R. Angew. Chem., Int. Ed. 2015, 54 12274-12296. doi:10.1002/anie.201503476

4. Grimme, S.; Schreiner, P. R. Angew. Chem., Int. Ed. 2011, 50, 12639-12642. doi:10.1002/anie.201103615

5. Schreiner, P. R.; Chernish, L. V.; Gunchenko, P. A.; Tikhonchuk, E. Y.; Hausmann, H.; Serafin, M.; Schlecht, S.; Dahl, J. E. P.; Carlson, R. M. K.; Fokin, A. A. Nature 2011, 477, 308-311. doi:10.1038/nature10367

6. Fokin, A. A.; Chernish, L. V.; Gunchenko, P. A.; Tikhonchuk, E. Yu.; Hausmann, H.; Serafin, M.; Dahl, J. E. P.; Carlson, R. M. K.; Schreiner, P. R. J. Am. Chem. Soc. 2012, 134, 13641-13650. doi:10.1021/ja302258q

7. Rösel, S.; Quanz, H.; Logemann, C.; Becker, J.; Mossou, E.; Cañadillas-Delgado, L.; Caldeweyher, E.; Grimme, S.; Schreiner, P. R. J. Am. Chem. Soc. 2017, 139, 7428-7431. doi:10.1021/jacs.7b01879

8. Aikawa, H.; Takahira, Y.; Yamaguchi, M. Chem. Commun. 2011, 47, 1479-1481. doi:10.1039/C0CC03025B

9. Hobza, P.; Müller-Dethlefs, K. Non-covalent Interactions; The Royal Society of Chemistry: Cambridge, 2009. doi:10.1039/9781847559906

10. Wheeler, S. E.; Seguin, T. J.; Guan, Y.; Doney, A. C. Acc. Chem. Res. 2016, 49, 1061-1069. doi:10.1021/acs.accounts.6b00096

11. Walden, D. M.; Ogba, O. M.; Johnston, R. C.; Cheong, P. H.-Y. Acc. Chem. Res. 2016, 49, 1279-1291. doi:10.1021/acs.accounts.6b00204

12. Daugulis, O.; Roane, J.; Tran, L. D. Acc. Chem. Res. 2015, 48, 1053-1064. doi:10.1021/ar5004626

13. Wencel-Delord, J.; Glorius, F. Nat. Chem. 2013, 5, 369-375. doi:10.1038/nchem.1607

14. Yeung, C. S.; Dong, V. M. Chem. Rev. 2011, 111, 1215-1292. doi:10.1021/cr100280d

15. Ackermann, L. Chem. Rev. 2011, 111, 1315-1345. doi:10.1021/cr100412j

16. Chen, X.; Engle, K. M.; Wang, D.-H.; Yu, J.-Q. Angew. Chem., Int. Ed. 2009, 48, 5094-5115. doi:10.1002/anie.200806273

17. Bergman, R. G. Nature 2007, 446, 391-393. doi:10.1038/446391a

18. Ghorai, D.; Müller, V.; Keil, H.; Stalke, D.; Zanoni, G.; Tkachenko, B. A.; Schreiner, P.; Ackermann, L. Adv. Synth. Catal. 2017, 359, 3137-3141. doi:10.1002/adsc.201700663

19. Graux, L. V.; Giorgi, M.; Buono, G.; Clavier, H. Dalton Trans. 2016, 45, 6491-6502. doi:10.1039/C5DT04683A

20. Zell, D.; Warratz, S.; Gelman, D.; Garden, S. J.; Ackermann, L. Chem. - Eur. J. 2016, 22, 1248-1252. doi:10.1002/chem.201504851

21. Ackermann, L.; Barfüsser, S.; Kornhaass, C.; Kapdi, A. R. Org. Lett. 2011, 13, 3082-3085. doi:10.1021/ol200986x

22. Ackermann, L.; Althammer, A.; Born, R. Angew. Chem., Int. Ed. 2006, 45, 2619-2622. doi:10.1002/anie.200504450

23. Ackermann, L. Org. Lett. 2005, 7, 3123-3125. doi:10.1021/ol051216e

24. Sperger, T.; Sanhueza, I. A.; Schoenebeck, F. Acc. Chem. Res. 2016, 49, 1311-1319. doi:10.1021/acs.accounts.6b00068

25. Ahlquist, M. S. G.; Norrby, P.-O. Angew. Chem., Int. Ed. 2011, 50, 11794-11797. doi:10.1002/anie.201105928

26. Hansen, A.; Bannwarth, C.; Grimme, S.; Petrović, P.; Werlé, C.; Djukic, J.-P. ChemistryOpen 2014, 3, 177-189. doi:10.1002/open.201402017

27. Lyngvi, E.; Sanhueza, I. A.; Schoenebeck, F. Organometallics 2015, 34, 805-812. doi:10.1021/om501199t

28. Ehrlich, S.; Moellmann, J.; Grimme, S. Acc. Chem. Res. 2013, 46, 916-926. doi:10.1021/ar3000844

29. Grimme, S. Wiley Interdiscip. Rev.: Comput. Mol. Sci. 2011, 1, 211-228. doi:10.1002/wcms.30 
30. Li, J.; Ackermann, L. Angew. Chem., Int. Ed. 2015, 54, 3635-3638. doi:10.1002/anie.201409247

31. Yu, D.-G.; Gensch, T.; de Azambuja, F.; Vásquez-Céspedes, S.; Glorius, F. J. Am. Chem. Soc. 2014, 136, 17722-17725. doi:10.1021/ja511011m

32. Pawar, A. B.; Chang, S. Org. Lett. 2015, 17, 660-663. doi:10.1021/ol503680d

33. Liu, W.; Ackermann, L. Chem. Commun. 2014, 50, 1878-1881. doi:10.1039/c3cc49502g

34. Cui, J.; Song, J.; Liu, Q.; Liu, H.; Dong, Y. Chem. - Asian J. 2018, 13, 482-495. doi:10.1002/asia.201701611

35. Grimme, S.; Antony, J.; Ehrlich, S.; Krieg, H. J. Chem. Phys. 2010, 132, 154104. doi:10.1063/1.3382344

36. Grimme, S.; Ehrlich, S.; Goerigk, L. J. Comput. Chem. 2011, 32, 1456-1465. doi:10.1002/jcc.21759

37. Krossing, I.; Raabe, I. Angew. Chem., Int. Ed. 2004, 43, 2066-2090. doi:10.1002/anie.200300620

38. Leitch, J. A.; Wilson, P. B.; McMullin, C. L.; Mahon, M. F.; Bhonoah, Y.; Williams, I. H.; Frost, C. G. ACS Catal. 2016, 6, 5520-5529. doi:10.1021/acscatal.6b01370

39. Kumar, N. Y. P.; Rogge, T.; Yetra, S. R.; Bechtoldt, A.; Clot, E.; Ackermann, L. Chem. - Eur. J. 2017, 23, 17449-17453. doi:10.1002/chem.201703680

40. Bu, Q.; Rogge, T.; Kotek, V.; Ackermann, L. Angew. Chem., Int. Ed. 2018, 57, 765-768. doi:10.1002/anie.201711108

41. Zell, D.; Dhawa, U.; Müller, V.; Bursch, M.; Grimme, S.; Ackermann, L. ACS Catal. 2017, 7, 4209-4213. doi:10.1021/acscatal.7b01208

42. Yang, Y.; Buchwald, S. L. Angew. Chem., Int. Ed. 2014, 53, 8677-8681. doi:10.1002/anie.201402449

43. Yang, Y.; Liu, P. ACS Catal. 2015, 5, 2944-2951. doi:10.1021/acscatal.5b00443

44. Orientating calculations using $\mathbf{2 b}$ and $\mathbf{2 c}$ as the cyanating agent parallel the trend observed in the kinetic data $\left(\Delta G^{\ddagger}(\mathbf{2} \mathbf{b})<\Delta G^{\ddagger}(\mathbf{2} \mathbf{b})<\right.$ $\left.\Delta G^{\ddagger}(\mathbf{2 c})\right)$.

45. Tao, J.; Perdew, J. P.; Staroverov, V. N.; Scuseria, G. E. Phys. Rev. Lett. 2003, 91, 146401. doi:10.1103/PhysRevLett.91.146401

46. Perdew, J. P.; Burke, K.; Ernzerhof, M. Phys. Rev. Lett. 1996, 77, 3865-3868. doi:10.1103/PhysRevLett.77.3865

47. Perdew, J. P.; Burke, K.; Ernzerhof, M. Phys. Rev. Lett. 1997, 78, 1396. doi:10.1103/PhysRevLett.78.1396

48. Johnson, E. R.; Keinan, S.; Mori-Sánchez, P.; Contreras-García, J.; Cohen, A. J.; Yang, W. J. Am. Chem. Soc. 2010, 132, 6498-6506. doi:10.1021/ja100936w

49. Contreras-García, J.; Johnson, E. R.; Keinan, S.; Chaudret, R.; Piquemal, J.-P.; Beratan, D. N.; Yang, W. J. Chem. Theory Comput. 2011, 7, 625-632. doi:10.1021/ct100641a

50. Wuttke, A.; Mata, R. A. J. Comput. Chem. 2017, 38, 15-23. doi:10.1002/jcc. 24508

51. Schneider, W. B.; Bistoni, G.; Sparta, M.; Saitow, M.; Riplinger, C.; Auer, A. A.; Neese, F. J. Chem. Theory Comput. 2016, 12, 4778-4792. doi:10.1021/acs.jctc.6b00523

52. Weigend, F.; Ahlrichs, R. Phys. Chem. Chem. Phys. 2005, 7, 3297-3305. doi:10.1039/b508541a

53. Becke, A. D. J. Chem. Phys. 1993, 98, 5648-5652. doi:10.1063/1.464913

54. Lee, C.; Yang, W.; Parr, R. G. Phys. Rev. B 1988, 37, 785-789. doi:10.1103/PhysRevB.37.785

55. Zhao, Y.; Truhlar, D. G. J. Chem. Phys. 2006, 125, 194101. doi:10.1063/1.2370993
56. Eichkorn, K.; Weigend, F.; Treutler, O.; Ahlrichs, R. Theor. Chem. Acc. 1997, 97, 119-124. doi:10.1007/s002140050244

57. Deglmann, P.; May, K.; Furche, F.; Ahlrichs, R. Chem. Phys. Lett. 2004, 384, 103-107. doi:10.1016/j.cplett.2003.11.080

58. Ribeiro, R. F.; Marenich, A. V.; Cramer, C. J.; Truhlar, D. G. J. Phys. Chem. B 2011, 115, 14556-14562. doi:10.1021/jp205508z

59. Klamt, A.; Schüürmann, G. J. Chem. Soc., Perkin Trans. 2 1993, 799-805. doi:10.1039/P29930000799

60. Werner, H.-J.; Knowles, P. J.; Knizia, G.; Manby, F. R.; Schütz, M. Wiley Interdiscip. Rev.: Comput. Mol. Sci. 2012, 2, 242-253. doi:10.1002/wcms.82

61. MOLPRO, version 2015.1; a package of ab initio programs, http://www.molpro.net.

62. Riplinger, C.; Sandhoefer, B.; Hansen, A.; Neese, F. J. Chem. Phys. 2013, 139, 134101. doi:10.1063/1.4821834

63. Riplinger, C.; Neese, F. J. Chem. Phys. 2013, 138, 034106. doi:10.1063/1.4773581

64. Liakos, D. G.; Neese, F. J. Chem. Theory Comput. 2015, 11, 4054-4063. doi:10.1021/acs.jctc.5b00359

65. Neese, F. Wiley Interdiscip. Rev. Comput. Mol. Sci. 2012, 2, 73-78. doi: $10.1002 / \mathrm{wcms} .81$

66. TURBOMOLE, V7.1, 2015; TURBOMOLE GmbH, since 2007, http://www.turbomole.com.

a development of University of Karlsruhe and Forschungszentrum Karlsruhe GmbH, 1989-2007.

67. Furche, F.; Ahlrichs, R.; Hättig, C.; Klopper, W.; Sierka, M.; Weigend, F. Wiley Interdiscip. Rev.: Comput. Mol. Sci. 2014, 4, 91-100. doi:10.1002/wcms.1162

68. Liu, W.; Richter, S. C.; Mei, R.; Feldt, M.; Ackermann, L. Chem. - Eur. J. 2016, 22, 17958-17961. doi:10.1002/chem.201604621 69. Sun, B.; Yoshino, T.; Matsunaga, S.; Kanai, M. Adv. Synth. Catal. 2014, 356, 1491-1495. doi:10.1002/adsc.201301110

\section{License and Terms}

This is an Open Access article under the terms of the Creative Commons Attribution License (http://creativecommons.org/licenses/by/4.0), which permits unrestricted use, distribution, and reproduction in any medium, provided the original work is properly cited.

The license is subject to the Beilstein Journal of Organic Chemistry terms and conditions:

(https://www.beilstein-journals.org/bjoc)

The definitive version of this article is the electronic one which can be found at: doi: $10.3762 /$ bjoc. 14.130 